\title{
Proyectiles de plomo en el antiguo Perú
}

Projectiles en plomb de l'ancien Pérou

Lead Sling Projectiles from Ancient Peru

William E. Brooks, Armando Farfán, Luisa Vetter Parodi y David Diestra

Lopez

\section{OpenEdition}

Journals

Edición electrónica

URL: http://journals.openedition.org/bifea/820

DOI: $10.4000 /$ bifea.820

ISSN: 2076-5827

\section{Editor}

Institut Français d'Études Andines

\section{Edición impresa}

Fecha de publicación: 1 abril 2013

Paginación: 13-23

ISSN: 0303-7495

\section{Referencia electrónica}

William E. Brooks, Armando Farfán, Luisa Vetter Parodi y David Diestra Lopez, « Proyectiles de plomo en el antiguo Perú », Bulletin de l'Institut français d'études andines [En línea], 42 (1) | 2013, Publicado el 01 agosto 2013, consultado el 05 noviembre 2020. URL : http://journals.openedition.org/bifea/820 ; DOI : https://doi.org/10.4000/bifea.820

\section{(c) (i) $\odot$}

Les contenus du Bulletin de l'Institut français d'études andines sont mis à disposition selon les termes de la licence Creative Commons Attribution - Pas d'Utilisation Commerciale - Pas de Modification 4.0 International. 


\title{
Proyectiles de plomo en el antiguo Perú
}

\author{
William E. Brooks* \\ Armando Farfán ${ }^{* *}$ \\ Luisa Vetter Parodi*** \\ David Diestra Lopez ${ }^{* * *}$
}

\section{Resumen}

Se han encontrado proyectiles de piedra con hondas (warak'as) en varios sitios arqueológicos en el Perú. Esto indica que esta arma fue comúnmente utilizada por sociedades precolombinas. La honda, denominada warak'a o huaraca en quechua, posee un amplio espectro espacial y temporal, siendo documentada desde hace muchos años en el Viejo y Nuevo Mundo y usada aún con fines pastoriles en el Perú rural de hoy. Tanto en el antiguo Perú como en el Viejo Mundo, la galena con plata (soroche), fue fundida para la obtención de la plata. Con un proceso diferente de la propia galena se obtenía el plomo. A causa de su facilidad de fundición, moldeo y peso, el plomo habría proporcionado munición bien formada y con masa consistente para la warak'a, lo que mejoraba su precisión de uso. Varios artefactos precolombino de plomo del antiguo Perú han sido simplemente identificados como lingotes o pesas; sin embargo, si prestamos atención a sus formas (bicónicas, ovoides o esféricas) (35 mm, 80-160 g), dimensiones (30-60 mm) y pesos (30-40 g), vemos cómo se asemejan a los proyectiles de plomo de las hondas antiguas de Europa.

Palabras clave: plomo, huaraca, warak'a, munición, proyectil

\section{Projectiles en plomb de l'ancien Pérou}

\section{Résumé}

Les projectiles en pierre qui ont été découverts sur divers sites archéologiques péruviens indiquent que la fronde (quechua, warak'a, huaraca; esp., honda) était en usage au Pérou avant la conquête

* Geólogo, Reston, VA 20191. E-mail: webgeology@aim.com

** Foreign Languages, George Mason University, Fairfax, VA 22030. E-mail: avfarfan12@aol.com

*** Estudios Andinos, Pontificia Universidad Católica del Perú, Lima. E-mail: luchivetter@hotmail.com

${ }^{* * * *}$ Metales, Museo Larco, Lima, Perú. E-mail: restaurodavid@hotmail.com 
espagnole. Arme ancestrale, très répandue dans l'Ancien monde, la fronde est encore utilisée dans de nombreuses zones rurales du pays, principalement pour rassembler des troupeaux d'animaux. Dans le Pérou antique, comme ailleurs dans l'Ancien monde, la galène argentifère (soroche) était un minerai exploité selon deux processus bien distincts pour sa teneur en argent et en plomb. Lourd et malléable, le plomb présente en outre un point de fusion peu élevé. Il permettait donc de façonner des projectiles de fronde de forme et de poids constants, favorisant des tirs d'une précision régulière sans précédent. Plusieurs objets péruviens en plomb datant d'avant la colonisation espagnole ont été identifiés comme des lingots ou des poids. Toutefois, il serait plus exact de les qualifier d'objets biconiques, ovoïdes (3060 mm, 30-40 g) ou sphériques (35 mm, 80-160 g). Par leur masse, leur forme et leurs dimensions autant que leur composition, ces objets péruviens en plomb ressemblent étonnamment aux balles de fronde retrouvées sur des champs de bataille de l'Europe antique, auxquelles ils sont assimilés dans le présent document.

Mots clés : plomb, balle, fronde, arme, projectile

\title{
Lead Sling Projectiles from Ancient Peru
}

\begin{abstract}
Ancient stone sling ammunition found at several archaeological sites in Peru indicates that the Ancient stone sling ammunition found at several archaeological sites in Peru. In ancient Peru, as in the Old World, argentiferous galena (soroche) was smelted to provide silver and in a distinctly different process, lead was smelted from galena for other uses. Because of its ease of smelting, casting, and weight, lead would have provided sling ammunition with consistent form and mass, and therefore, improved and consistent accuracy. Several pre-contact lead artifacts from ancient Peru have been described as bars or weights; however, alternatively, these artifacts might also be more accurately described as biconic to ovoid (30-60 mm, 30-40 g) and spherical (35 mm, 80-160 g). In weight, form and dimensions, and composition, these ancient Peruvian lead artifacts are strikingly similar to ancient lead sling projectiles from ancient battle sites in Europe and are herein interpreted to have served a similar function.
\end{abstract}

Key words: lead, projectile, sling, weapon, ammunition

\section{INTRODUCCIÓN}

... con una honda y una piedra, el joven David mató al gigante filisteo (1 Samuel 17:40-49).

La honda es un arma antigua de largo alcance conocida por haber sido utilizada en diferentes partes del mundo (Korfmann, 1973; York \& York, 2011) y su lugar en la historia está asegurado por el combate entre David, levemente armado, y Goliat, equipado para una batalla. Con habilidad excepcional, con su honda y un canto rodado de río, David derribó a Goliat quien, inconsciente, fue después decapitado.

Las warak'as u hondas rara vez se conservan en registros arqueológicos debido a que los materiales con los que eran hechas (tela, cuerda o cuero) son de fácil descomposición. Sin embargo, en Perú, se ha hallado una honda de tela en la mina Primavera (Intermedio temprano [200-600 a. C.]) (Vaughn et al., 2012: 169) 
y para el Viejo Mundo se han hallado hondas en la tumba de Tutankhamen quien murió en el año 1325 a. C. y estas aparecen en escenas de batalla en Asiriao y en bajorrelieves romanos, en monedas y en tapices. La honda también fue descrita por Homero, pero lo que es más importante aún, para esta investigación, es que Korfmann (1973) señala que una compañía de 200 soldados de Rodas utilizando hondas y proyectiles de plomo tuvo dos veces el alcance de sus adversarios persas que utilizaron proyectiles de piedra. También indica que un hondero que utiliza munición de plomo podría alcanzar una trayectoria de más de 400 metros. En el mundo antiguo, los honderos de las Baleares, célebres por su precisión, llevaban tres hondas en la batalla: una honda de corto alcance, una honda de alcance intermedio para el segundo nivel y una honda más larga para disparos de largo alcance. La munición de la honda incluía cantos rodados de río, arcilla secada al sol o plomo. Sin embargo, la munición de plomo empezó a ser utilizada en la época clásica griega.

En Grecia, el plomo estuvo disponible en muchos sitios, por ejemplo, en Laurion, Mykonos y Thera (Healy, 1978; Higgins \& Higgins, 1996). La masa del plomo y la facilidad de lanzarlo en tamaños normales habría hecho del plomo un material superior y mortal como munición. Korfmann (1973: 40) presenta una ilustración que muestra un molde de arcilla, hallado en un lugar en Grecia, que fue utilizado para moldear proyectiles de plomo.

Algunos de los proyectiles de plomo del Viejo Mundo llevan diseños, nombres o insultos para la víctima. Era importante que el proyectil tuviera un sitio muy seguro en la honda y la forma bicónica o esférica era la preferida. El peso medio de munición de la honda era de aproximadamente 25 gramos; sin embargo, el proyectil de plomo utilizado por honderos baleares era de casi 185 g (Korfman, 1973).

\section{LAS WARAK'AS Y MUNICIONES EN LOS ANDES}

El arma más efectiva de largo alcance en el antiguo Perú fue la warak'a (honda) siendo el arma incaica más temida por los europeos. Sin embargo, la warak'a se utilizaba para lanzar proyectiles al enemigo y causar incendios en los techos de paja (Galimberti, 1951: 101; Howard, 1968: 101; Fernández Baca, 1989: 198, 218). En los años tempranos de 1500, un soldado español describió la destreza de los huaraqueros incaicos y escribió:

... he visto una piedra lanzada por una honda que podría matar un caballo o romper una espada en dos pedazos... (De Zárate, 1968 [1555]: 164).

Pero, a veces la importancia y el uso de la honda han sido casi olvidados (Dohrenwend, 2002; York \& York, 2011). Por ejemplo, en su investigación sobre las armas de metal, Pérez Maestro (1999) no ofrece datos sobre el uso de la warak'a como un arma incaica, ni tampoco sobre proyectiles de plomo. Ghezzi (2007: 6) indica que «... la metalurgia tuvo un impacto limitado en la tecnología bélica»; en los Andes tampoco hay datos sobre proyectiles de plomo. Esta investigación es la primera en preguntar y establecer el uso de proyectiles de plomo como 
munición de warak'a en el Perú prehispánico; de igual manera fueron utilizadas como munición de honda en el Viejo Mundo.

Normalmente, con la warak'a u honda se usaba munición de piedra, disponible en ríos y quebradas locales. En investigaciones en sitios arqueológicos del norte del Perú, como Cerro La Cruz, se documentaron y clasificaron piedras del calibre de un puño, que fueron interpretadas como munición (Topic, 1990). La presencia de esta munición en estos sitios, como Cerro La Cruz, confirma el uso de la warak'a en los antiguos Andes. Hoy en día, en el Puno rural, la warak'a se usa con gran amplitud (Harrison, 2011) pero con la finalidad de pastar animales. Brown Vega \& Craig (2009) han demostrado que un tiro de la warak'a, utilizando la munición de piedra, varía entre $50 \mathrm{~m}$ y $140 \mathrm{~m}$.

\section{EL PLOMO EN EL ANTIGUO PERÚ}

El oro, la plata y el cobre son los metales más conocidos del Perú moderno y antiguo; sin embargo, el Perú actualmente produce un promedio de 300000 toneladas métricas de plomo anuales, principalmente de yacimientos de plomo-zinc en el Perú central (Gurmendi, 2010, Tabla 1: 17.8; Ministerio de Energía y Minas, 2000; Ministry of Energy and Mines, 1999). El plomo rara vez se presenta como metal nativo; por lo tanto, es importante establecer que el plomo, tanto como la galena $[\mathrm{PbS}]$, se obtuvieron de minas en el antiguo Perú. Petersen (2010 [1970]: 30) da descripciones de varios artefactos precolombino de plomo, algunos de los cuales los atribuye a la cultura Moche (100 a. C.700 d. C.), lo cual confirma la minería y fundición del plomo durante épocas precolombino.

Artefactos precolombino de plomo del Perú forman parte de colecciones arqueológicas en Berlín, Alemania; el Museo Bruning, Lambayeque, Perú; el Museo Amano y el Museo Larco en Lima (Petersen, 2010 [1970]: 30); y el Museo de América, Madrid (Cuesta, 1980: 317). El catálogo en línea del Museo Larco, Lima, Perú, incluye 17 artefactos precolombinos de plomo descritos como lingotes, pesas y miniaturas esculturas (Museo Larco, s. f.).

Cerca de la fundición actual de plomo-zinc-cobre de La Oroya, en el Valle del Mantaro (Perú central) (Wells, 1940) se encontraron óxido de plomo, esferas y otros artefactos de plomo en el lugar «Sitio C» concluyendo que la fundición del plomo había tenido lugar antes de la llegada de los españoles. Howe \& Petersen (1994) describieron 23 artefactos de plomo que incluían esferas, pesas, y tiras dobladas de un sitio preinka-Inka, también en el Valle del Mantaro. Sin embargo, en los ejemplos antes mencionados, no se incluyeron detalles como el peso o las dimensiones de los objetos. No se describió una ranura en las piezas - esta observación es importante, porque la presencia de una ranura habría servido para conectar el peso de plomo a una cuerda- y por lo tanto, la ausencia de una ranura u otra característica de fijación lleva a considerar otros usos para dichos artefactos. 
En el año 2009 se documentó un fragmento de plomo doblado de $2 \times 3 \mathrm{~cm}$, en el sitio arqueológico El Purgatorio, cerca de Casma (Melissa Vogel, arqueóloga, Universidad de Clemson, South Carolina, comunicación escrita, el 11 de agosto de 2011) y hay afloramientos de plomo-zinc cerca al sitio (Sánchez et al., 1995: 211). En otro lugar del norte del Perú, se han levantado mapas de la presencia de zinc-plata-plomo cerca de Buldibuyo (Wilson et al., 1967).

Petersen (2010 [1970]: 31) proporciona un análisis químico de un vaso de plomo de La Ventana, un sitio Chimú (1100-1450 a. C.) cerca de Lambayeque. El análisis indica que se compone de un $98,7 \%$ de plomo (por ejemplo, la ley de plomo de un horno de fundición es de 99 \% de plomo; Hofman, 1918: 13) y un $<0,01 \%$ de plata siendo este dato consecuente con la existencia de la producción de plomo como material primario para el uso industrial de la galena no argentífera y que el plomo no fue necesariamente un subproducto de la producción de la plata (Howe \& Petersen, 1994).

Los estudios geoquímicos y geocronológicos de los sedimentos de los lagos cerca de Cerro de Pasco, una mina de zinc-plata-plomo localizada en el Perú central, indica que el enriquecimiento de plomo en los sedimentos a causa de la fundición del metal empezó cerca del 600 d. C., es decir mucho antes de la llegada de los europeos (Cooke et al., 2009).

\section{ANTIGUA FUNDICIÓN DE PLOMO}

Desde el siglo XVI, el sitio Inka de Curamba, departamento de Apurímac, en el sur del Perú, ha sido interpretado como un centro metalúrgico para procesar mena de plata. Sin embargo, aparte de las muchas depresiones u hornos superficiales, no había pruebas materiales como fragmentos u otras indicaciones del uso del $\sim 1 \mathrm{~m}$ huayras o tocochimbos de piedra o estructuras de arcilla tradicionalmente utilizadas para la fundición de plata en las culturas de los Andes (Petersen, 2010 [1970]: 47; Van Buren \& Mills, 2005).

Ensayos geoquímicos (Inductively Coupled Plasma o Plasma Acoplado Inductivamente) de las escorias excavadas del horno de hoyos de fundición en Curamba indicaban un nivel bajo de plata $(<0,3$ a $0,4 \mathrm{ppm})$, de oro $(<2 \mathrm{ppm})$ y de cobre (18 a 31 ppm); mientras que el contenido de plomo (155 a 234 ppm) y zinc (125 a 259 ppm) era relativamente alto. Por ejemplo, una muestra de galena con plata o sorojche (también llamada galena sucia) de Porco, Bolivia, contenía 3250 ppm Ag (Brooks \& Van Buren, 2007).

Gracias a la técnica de espectroscopia de Mössbauer, aplicada a algunas muestras de los hornos de Curamba, se sabe que alcanzaron una temperatura máxima de $800 \pm 50{ }^{\circ} \mathrm{C}$ (Vetter Parodi et al., 2008). Esta temperatura es más que adecuada para fundir plomo $\left(327^{\circ} \mathrm{C}\right)$. Hasta ahora, Curamba es el horno de fundición de plomo mejor descrito en el antiguo Perú (Vetter Parodi et al., 2008; Brooks \& Vetter Parodi, 2012). 


\section{MUESTRAS}

Las descripciones físicas y los dibujos de proyectiles de plomo del Viejo Mundo también han sido proporcionados por Korfmann (1973), Savage (1999) y Contreras et al. (2006). En las figuras 1 y 2 se muestran ejemplos de proyectiles de honda hechos de plomo, tipo bicónico, provienentes de campos de batalla en el Viejo Mundo. Estas muestras son utilizadas para fines de comparación forense con los artefactos del Museo Larco, en la figura 3, que habían sido descritos anteriormente como lingotes y pesas de plomo:
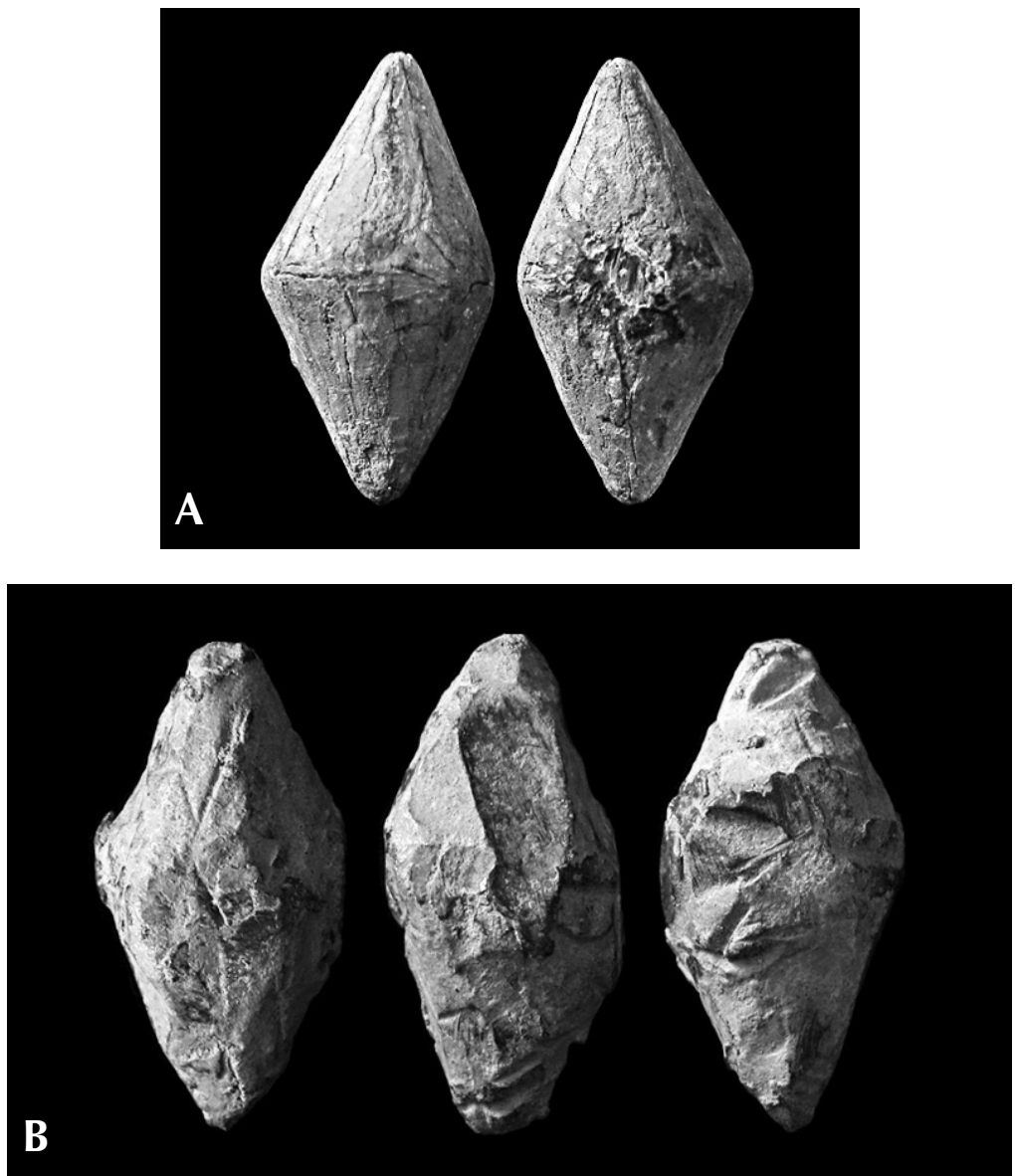

Figura 1 - A) Proyectil de plomo que se encontró en un campo de batalla celtaromano del siglo I a. C., en España, $39 \mathrm{~mm} / 40 \mathrm{~g}$; B) Proyectil de plomo del siglo I a. C., inscrito con una $\mathrm{V}$ para la Legión $\mathrm{V}$ (la legión quinta de Julio César), encontrado en un campo de batalla romano cerca de Córdoba, España, con cicatrices del daño de batalla, 36 mm/37 g (Cortesía de Gabriel Vandevort (15 de noviembre de 2011).

Fuente: http://www.ancientresource.com/lots/roman/weapons-sling-bolts.html 
Proyectiles de plomo en el antiguo Perú
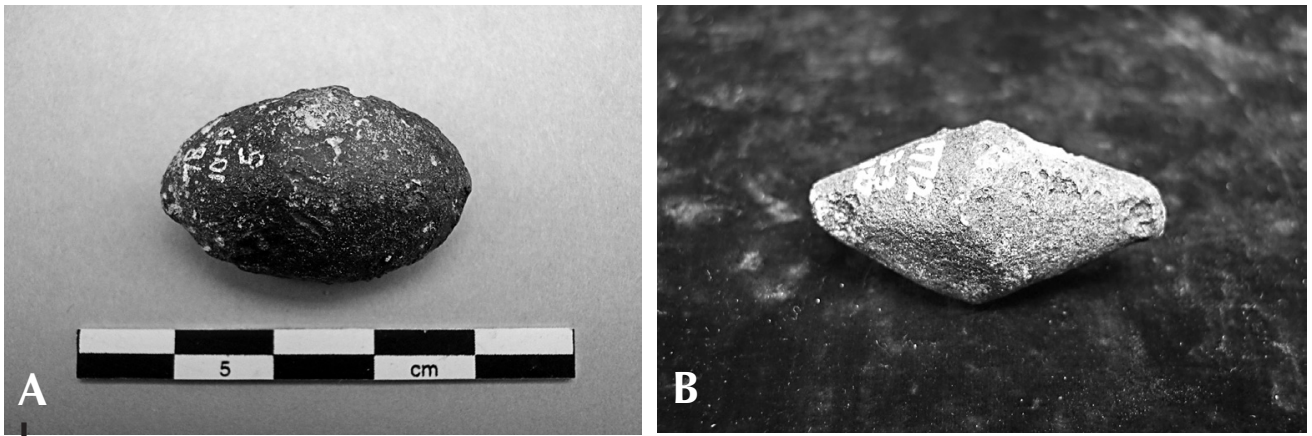

Figura 2 - A) Proyectil de plomo para honda, de forma almendrada, número de registro 1878.1019.51 $(30 \mathrm{~mm} / 35.7 \mathrm{~g})$; B) Proyectil de plomo para honda, de forma octaédrica, número de registro 1772.0303-127 (37 mm/50 g)

Antigüedades griegas y romanas, donada por el Teniente General Augustus Meyrick (1878), fotografía (C) Trustees of The British Museum, el 11 de marzo de 2012

Fuente: http://www.britishmuseum.org/
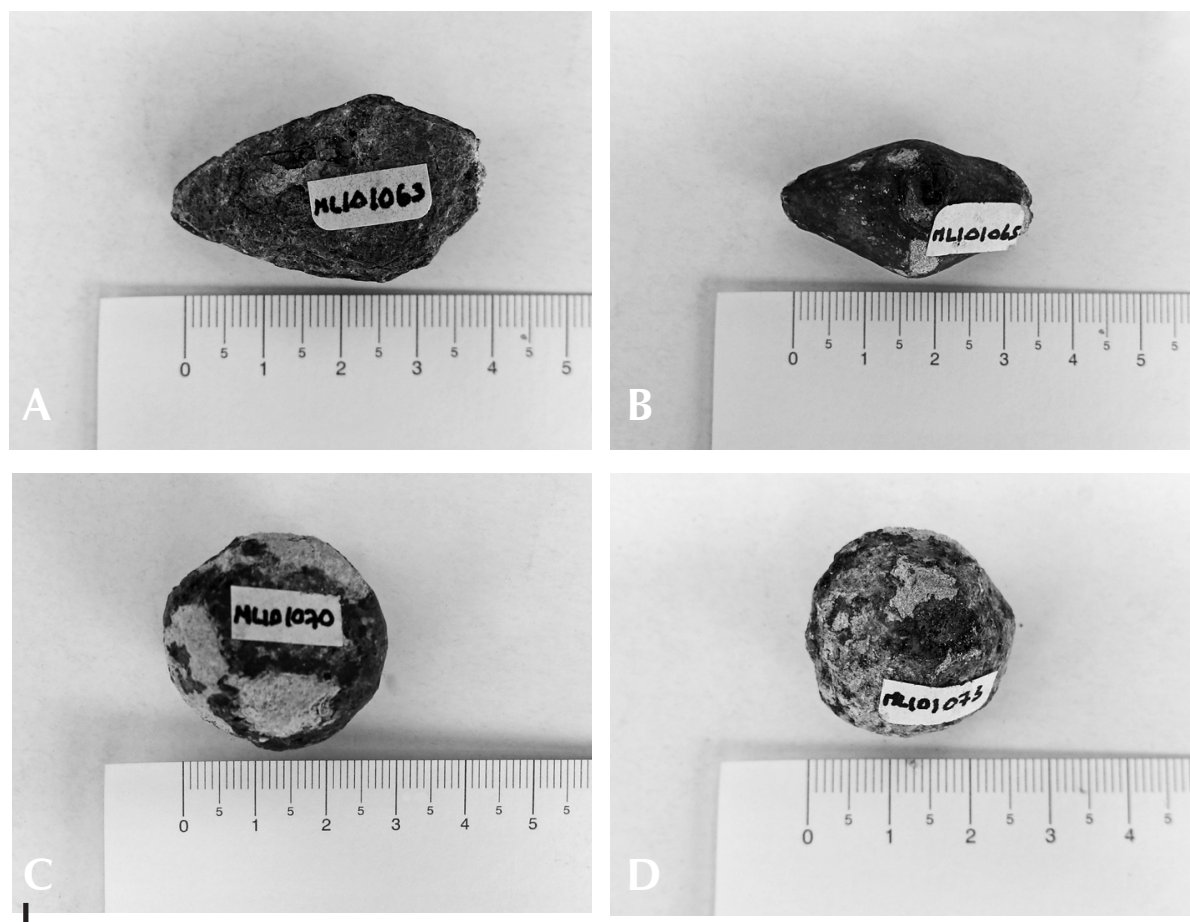

Figura 3 - Artefactos de plomo: A) ML101063 (57 mm/36 g); B) ML101065 (34 mm/43 g); C) ML101070 (28 mm/110 g); D) ML101073 (24 mm/60 g)

Años 1476-1532 d. C., Chimú-Inca, costa norte, cronología por John Rowe (Museo Larco, Lima-Perú, 9 de noviembre de 2011)

Fuente: http://www.museolarco.org/ 


\section{1. Peso}

Los proyectiles de plomo (figs. 1 y 2), y los descritos por Korfmann (1973), pesan de 12 a $39 \mathrm{~g}$, sin embargo proyectiles más pesados, de 30 a $35 \mathrm{~g}$, han sido descritos (Korfmann, 1973). La galena, la mena de plomo [PbS], es un mineral pesado y denso y también puede incluir cantidades secundarias de plata, arsénico o cobre. La galena tiene una gravedad específica de 7,6; sin embargo, el plomo tiene una gravedad específica más alta, de 11,3 y, como dato comparativo, el oro nativo tiene una gravedad específica mucho más alta de 15,5 a 19,3.

\section{2. Dimensiones y forma}

Las muestras de proyectiles (figs. 1 y 2) y los dos artefactos de plomo del Museo Larco (fig. 3 A y B) son bicónico-ovoides. Esto habría proporcionado un asiento seguro dentro de la bolsa de la warak'a. Esta forma bicónica puede haber sido más aerodinámica y muy similar a un balón de fútbol americano. Un croquis de un proyectil estilo bicónico seguramente sentado en una warak'a es presentado por Harrison (2011). También se han descrito formas esféricas. La dimensión más grande de los ejemplares de estilo bicónico es de 36 a 39 mm y la dimensión más grande de los artefactos del Museo Larco es típicamente de 30 a $42 \mathrm{~mm}$. El diámetro de las formas esféricas del Museo Larco (fig. 3 C y D) era 24 a 28 mm.

\section{3. Composición}

Las muestras (figs. 1 y 2) y los artefactos del Museo Larco (fig. 3) están hechos de plomo. Este metal habría proporcionado una masa de inercia más alta (la resistencia de un objeto a su cambio en la velocidad), y por lo tanto, mayor energía cinética (la energía que posee debido a su movimiento) para un impacto más mortal.

En el siglo I a. C., Onasandrius (Gray, 2008) escribió en su libro Estrategia «... que la honda es una arma mortal utilizada por la infantería y puesto que el plomo es el mismo color como el cielo, la proyectil no es visible, el impacto imprevisible y duro, penetrando la proyectil profundamente en el cuerpo». En su tratado De Medicina, Celsus ( 25 a. C.) (Bruce, 2001), un científico romano, describe técnicas quirúrgicas para extraer proyectiles de plomo y piedra de soldados heridos.

Basado en la narrativa del soldado español, que cuenta que un huaraquero inca podría matar un caballo (De Zárate, 1968 [1555]: 164), es lógico inferir que un huaraquero también podría matar a un hombre. La fuerza de ese impacto mortal puede ser cuantificada en joules con la ecuación $\mathrm{KE}=1 / 2 \mathrm{MV}^{2}$ y utilizar una masa $(\mathrm{M})$ de $40 \mathrm{~g}(0,04 \mathrm{~kg})$ y una velocidad (V) de $45 \mathrm{~m} / \mathrm{sec}(160 \mathrm{~km} / \mathrm{h})$ (Savage, 1999: 13):

$\mathrm{KE}=1 / 2 \mathrm{MV}^{2}$

$\mathrm{KE}=1 / 2(0.04 \mathrm{~kg}) \times(45 \mathrm{~m} / \mathrm{sec})^{2}$

$\mathrm{KE}=40,5$ joules 
Como dato comparativo, una bala de calibre .45, que está compuesta típicamente de una aleación de plomo-antimonio, pesa solamente $15 \mathrm{~g}$ y tiene un diámetro de $11,5 \mathrm{~mm}$, es más pequeña que los proyectiles de las figuras, sin embargo, la energía cinética de una bala calibre .45 excede claramente la del proyectil (bala) de plomo de la warak'a debido al cambio extremo de velocidad.

\section{CONCLUSIÓN}

El uso de la warak'a y munición de piedra en el antiguo Perú ha sido documentado anteriormente por arqueólogos e historiadores como Galimberti (1951), Howard (1968), Fernández Baca (1989), Topic (1990) y Ghezzi (2007). Sin embargo, esta investigación indica la disponibilidad del plomo (como galena) y el uso de proyectiles de plomo en el Perú antiguo, y lo que es más importante, las similitudes físicas entre los proyectiles de plomo del Viejo Mundo y varios ejemplos de artefactos (precolombino) de plomo del Museo Larco, Lima, Perú. Esta comparación de la composición, el peso, la forma y las dimensiones de los ejemplares del Viejo Mundo con los artefactos de plomo precolombino del Museo Larco indican que los huaraqueros del Perú antiguo entiendían, como sus contrapartes del Viejo Mundo, las ventajas de los proyectiles de plomo en el campo de batalla.

\section{Referencias citadas}

BROOKS, W. E. \& VAN BUREN, M., 2007 - Dirty galena at Porco, Bolivia. Geological Society of America Abstracts with Programs, 39 (6): 549.

BROOKS, W. E. \& VETTER PARODI, L., 2012 - Antigua fundición de plomo en el sitio inca de Curamba, departamento de Apurímac, Perú. Bulletin de l'Institut Français d'Études Andines, 41 (2): 197-208.

BROWN VEGA, M. \& CRAIG, N., 2009 - New experimental data on the distance of sling projectiles. Journal of Archaeological Science, 36 (6): 264-1268.

BRUCE, D., 2001 - Aulus Cornelius Celsus biography (c. 25 BC-50 BC). Disponible en: http://www.faqs.org/health/bios/65/Aulus-Cornelius-Celsus.htm (acceso realizado el 30 de noviembre de 2012).

CONTRERAS, F., MÜLLER, R. \& VALLE, F. J., 2006 - El asentamiento militar romano de Sanitija (1234-1245): una aproximación a su contexto histórico. Mayurqa, 31: 231 249.

COOKE, C. A., WOLFE, A. P. \& HOBBS, W. O., 2009 - Lake-sediment geochemistry reveals 1400 years of evolving extractive metallurgy at Cerro de Pasco, Peruvian Andes. Geology, 37 (2): 1019-1022.

CUESTA, M., 1980 - Arqueología Andina-Perú, 370 pp.; Madrid: Museo de América, Ministerio de Cultura, Dirección del Patrimonio Artístico, Archivos y Museos. 
DE ZÁRATE, A., 1968 [1555] - The discovery and conquest of Peru, 210 pp.; Nueva York: Penguin Books.

DOHRENWEND, R. E., 2002 - The sling, forgotten firepower of antiquity. Journal of Martial Arts, 11: 28-49.

FERNÁNDEZ BACA, J., 1989 - Motivos de ornamentación de la cerámica Inca Cusco, 250 pp.; Cusco: Editorial Navarrete.

GALIMBERTI, C. A., 1951 - Las armas de guerra Incaicas y su evolución. Revista del Museo e Instituto Arqueológico, 13-14: 89-120; Cusco: Universidad Nacional del Cusco.

GHEZZI, I., 2007 - La naturaleza de la guerra prehispánica. Revista Andina, 44: 199-226.

GRAY, T. W., 2008 - Roman sling bullets. Disponible en: http://periodictable.com/ Items/082,58/index.htr (consulta realizada el 30 de noviembre de 2012).

GURMENDI, A. C., 2010 - The mineral industry of Peru; U.S. Geological Survey Minerals Yearbook, 3: 15.1-15.14. Disponible en: http://minerals.usgs.gov/minerals/pubs/ country/2010/myb3-2010-pe.pdf

HARRISON, C., 2011 - Welcome to slinging. Disponible en: http://www.slinging.org

HEALY, J. F., 1978 - Mining and metallurgy in the Greek and Roman World, 316 pp.; Londres: Thames and Hudson.

HIGGINS, M. D. \& HIGGINS, R., 1996 - A geological companion to Greece and the Aegean, 240 pp.; Nueva York: Cornell University Press

HOFMAN, E. M., 1918 - Metallurgy of lead, 664 pp.; Nueva York: McGraw-Hill.

HOWARD, C., 1968 - Pizarro and the conquest of Peru, 400 pp.; New York: American Heritage Publishing Co.

HOWE, E. \& PETERSEN, U., 1994 - Silver and lead in late prehistory of the Mantaro Valley, Peru. In: Archaeometry of Pre-Columbian Sites and Artifacts (D. A. Scott \& P. Meyers, eds.): 183-197; Los Ángeles: UCLA, Institute of Archaeology and the Getty Conservation Institute.

KORFMANN, M., 1973 - The sling as a weapon. Scientific American, 229 (4): 36-42.

MINISTERIO DE ENERGÍA Y MINAS, 2000 - Atlas de la pequeña minería en el Perú 2000; Lima: Ministerio de Energía y Minas, Dirección General de Minería.

MINISTRY OF ENERGY AND MINES, 1999 - Atlas. Mining and energy in Perú, 111 pp.; Lima: Ministry of Energy and Mines.

MUSEO LARCO, S. F. - Tesoros del antiguo Perú, plomo; Lima: Museo Larco. Disponible en: http://www.museolarco.org/

PÉREZ MAESTRO, C., 1999 - Armas de metal en el Perú prehispánico. Espacio, Tiempo y Forma, Serie II, Prehistoria y Arqueología, t. 12: 319-346.

PETERSEN, G., 2010 [1970] - Mining and metallurgy in ancient Perú. A translation of Minería y Metalurgia en el Antiguo Perú, 90 pp.; Colorado: Geological Society of America.

SÁNCHEZ, A., MOLINA, O. \& GUTIÉRREZ, R., 1995 - Geología de los cuadrángulos de Chimbote, Casma, y Culebras, hojas 19f, 19g, 20g, 263 pp.; Lima: Instituto Geológico Minero y Metalúrgico.

SAVAGE, C., 1999 - The sling for sport and survival, 64 pp.; Port Townsend, WA: Loompanics Unlimited.

TOPIC, T. L., 1990 - Territorial expansion and the Kingdom of Chimor. In: The Northern Dynasties, Kingship and Statecraft in Chimor (M. E. Moseley \& A. Cordy-Collins, eds.): 177-194; Washington, DC: Dumbarton Oaks Research Library.

VAN BUREN, M. \& MILLS, B. H., 2005 - Huayrachinas and tocochimbos, traditional smelting technology of the southern Andes. Latin American Antiquity, 16 (1): 3-25. 
VAUGHN, K. J., VAN GIJSEGHEM, H., WHALEN, V. H., EERKENS, J. W. \& LINARES G. M., 2012 - The organization of mining in Nasca during the early intermediate period: Recent evidence from Mina Primavera. In: Mining and Quarrying in the Ancient Andes (N. Tripcevich \& K. J. Vaughan, eds.): 157-182; New York: Ancient Andes, Interdisciplinary Contributions to Archaeology, Springer Publishing. doi 10.1007/978-1 4614-5200-3_10

VETTER PARODI, L., PETRICK CASAGRANDE, S., HUAYPAR VÁSQUEZ, Y. \& MAC KAY FULLE, M., 2008 - Los hornos metalúrgicos del sitio Inca de Curamba, Perú. Bulletin de l'Institut Français d'Études Andines, 37 (3): 451-475.

WELLS, R. J., 1940 - A superficial inspection of archaeological sites near La Oroya, Peru. Actas y Trabajos Científicos del XXXII Congreso Internacional de Americanistas, Lima, 1939, T. I: 347-354.

WILSON, J., REYES, L. \& GARAYAR, J., 1967 - Geología de los cuandrángulos de Mollebamba, Yatabamba, Tayabamba, y Carhuaz, 96 pp.; Lima: Ministerio de Energía y Minas, Dirección General de Minería.

YORK, R. \& YORK, G., 2011 - Slings and slingstones, forgotten weapons of Oceania and the Americas, 224 pp.; Ohio: Ohio Kent State University Press. 